\title{
SELF-SIMILAR SOLUTIONS OF CURVATURE FLOWS IN WARPED PRODUCTS
}

\author{
SHANZE GAO AND HUI MA
}

\begin{abstract}
In this paper we study self-similar solutions in warped products satisfying $F-\mathcal{F}=\bar{g}\left(\lambda(r) \partial_{r}, \nu\right)$, where $\mathcal{F}$ is a nonnegative constant and $F$ is in a class of general curvature functions including powers of mean curvature and Gauss curvature. We show that slices are the only closed strictly convex self-similar solutions in the hemisphere for such $F$. We also obtain a similar uniqueness result in hyperbolic space $\mathbb{H}^{3}$ for Gauss curvature $F$ and $\mathcal{F} \geq 1$.
\end{abstract}

\section{INTRODUCTION}

Self-similar solutions are important in the study of mean curvature flow and powers of Gauss curvature flow in Euclidean space, since they describe the asymptotic behaviors near the singularities (See $[11,7,4,10]$ etc). Remarkable results due to Huisken [11] and Choi-Daskalopoulos [6], Brendle-Choi-Daskalopoulos [5] show the uniqueness of closed self-similar solutions for mean curvature flows and powers of Gauss curvature flows respectively. Although relation between self-similar solutions of general curvature flows and their singularities is unclear now, there have been some study on rigidity of closed self-similar solutions of curvature flows, for instance, [12], [9], etc. Recently self-similar solutions of the mean curvature flows were introduced on manifolds endowed with a conformal vector field [1], such as Riemannian cone manifolds [8] and warped product manifolds [14, 1]. In this paper we study closed strictly convex self-similar solutions of a class of curvature flows in Riemannian warped products and obtain the uniqueness of closed strictly convex self-similar solutions in hemispheres and hyperbolic spaces.

Let $N=[0, \bar{r}) \times \mathbb{S}^{n}$ be a warped product with metric $\bar{g}=d r^{2}+\lambda^{2}(r) g_{\mathrm{s}}$, where $\lambda$ is a positive warping factor and $g_{\mathrm{s}}$ is the standard metric of $\mathbb{S}^{n}$. Let $X: M \rightarrow N$ be a smooth embedding of a closed hypersurface in $N$ with $n \geq 2$, satisfying the following equation

$$
F(\kappa(x))-\mathcal{F}=\bar{g}\left(\lambda(r(x)) \partial_{r}(x), \nu(x)\right),
$$

for all $x \in M$, where $\mathcal{F}$ is a constant which can be regarded as a forcing term with respect to the flow, $\nu$ is the outward unit normal vector field of $M$ and $F$ is a homogeneous smooth symmetric function of the principal curvatures $\kappa=\left(\kappa_{1}, \kappa_{2}, \ldots, \kappa_{n}\right)$ of $M$, which satisfies the following condition.

Condition 1.1. Suppose $F$ is a smooth function defined on the positive cone $\Gamma_{+}=$ $\left\{\kappa \in \mathbb{R}^{n} \mid \kappa_{1}>0, \kappa_{2}>0, \cdots, \kappa_{n}>0\right\}$ of $\mathbb{R}^{n}$, and satisfies the following conditions:

2010 Mathematics Subject Classification. 53C44, 53C40.

Key words and phrases. self-similar solution, warped product.

This work was supported by National Natural Science Foundation of China (Grant No. 11671223). 
i) $F$ is positive and strictly increasing, i.e., $F>0$ and $\frac{\partial F}{\partial \kappa_{i}}>0$ for $1 \leq i \leq n$.

ii) $F$ is homogeneous symmetric function with degree $\beta$, i.e., $F(t \kappa)=t^{\beta} F(\kappa)$ for all $t \in \mathbb{R}_{+}$.

iii) For any $i \neq j$,

$$
\frac{\frac{\partial F}{\partial \kappa_{i}} \kappa_{i}-\frac{\partial F}{\partial \kappa_{j}} \kappa_{j}}{\kappa_{i}-\kappa_{j}} \geq 0
$$

iv) For all $\left(y_{1}, \ldots, y_{n}\right) \in \mathbb{R}^{n}$,

$$
\sum_{i} \frac{1}{\kappa_{i}} \frac{\partial \log F}{\partial \kappa_{i}} y_{i}^{2}+\sum_{i, j} \frac{\partial^{2} \log F}{\partial \kappa_{i} \partial \kappa_{j}} y_{i} y_{j} \geq 0 .
$$

We know that $\lambda(r) \partial_{r}$ is a conformal vector field on $N$ and $\bar{\nabla}_{Y}\left(\lambda \partial_{r}\right)=\lambda^{\prime} Y$ for any vector field $Y$ on $N$. For a warped product $N$, when the warping factor $\lambda(r)=r$, $\sin r$, or $\sinh r, N$ is the Euclidean space $\mathbb{R}^{n+1}$, the sphere $\mathbb{S}^{n+1}$ or the hyperbolic space $\mathbb{H}^{n+1}$ with constant sectional curvature $\epsilon=0,1$ or -1 respectively. In $\mathbb{R}^{n+1}$, $\lambda(r) \partial_{r}$ is just the position vector. So in the spirit of $[14,1]$, we call solutions of (1.1) self-similar solutions to the following curvature flow

$$
\frac{\partial}{\partial t} \tilde{X}=-(F-\mathcal{F}) \nu
$$

We give a further brief explanation here and more details can be found in [1]. If $\tilde{X}$ satisfies the equation

$$
\frac{\partial}{\partial t} \tilde{X}=-\phi(t) \lambda \partial_{r}
$$

for a smooth function $\phi(t)$ on $t$, then it gives a family of conformal hypersurfaces. Suppose $\tilde{X}$ satisfies (1.3) and (1.4) simultaneously, then $\tilde{X}$ satisfies

$$
F-\mathcal{F}=\phi(t) \bar{g}\left(\lambda \partial_{r}, \nu\right)
$$

up to a tangential diffeomorphism for each $t \in[0, T)$. This is why solutions to (1.1) are called self-similar solutions to (1.3).

In this paper, we prove the following main theorem.

Theorem 1.2. Let $M$ be a closed, strictly convex hypersurface in the hemisphere $\mathbb{S}_{+}^{n+1}$ satisfying

$$
F-\mathcal{F}=\bar{g}\left(\lambda \partial_{r}, \nu\right)
$$

For $\beta \geq 1$ and $\mathcal{F} \geq 0$, if $F$ satisfies Condition 1.1 , then $M$ is a slice $\left\{r_{0}\right\} \times \mathbb{S}^{n}$ in $\mathbb{S}_{+}^{n+1}$.

Remark 1.3. In Euclidean space, a similar theorem is proven for $\beta>1$ in [9]. Due to the positivity of sectional curvature, we can achieve $\beta=1$ for the hemisphere.

Let

$$
\sigma_{k}(\kappa)=\sum_{1 \leq i_{1}<i_{2} \cdots<i_{k} \leq n} \kappa_{i_{1}} \kappa_{i_{2}} \cdots \kappa_{i_{k}}, \quad S_{k}(\kappa)=\sum_{i=1}^{n} \kappa_{i}^{k},
$$

be the $k$-th elementary symmetric function and the $k$-th power sum of principal curvatures, respectively. Since $\sigma_{k}^{\alpha}$ and $S_{k}^{\alpha}$ satisfy Condition 1.1 if $\alpha>0$ (see [9]), we have the following corollaries immediately. 
Corollary 1.4. Let $M$ be a closed, strictly convex hypersurface in the hemisphere $\mathbb{S}_{+}^{n+1}$ satisfying

$$
\sigma_{k}^{\alpha}(\kappa)-\mathcal{F}=\bar{g}\left(\lambda \partial_{r}, \nu\right) .
$$

If $1 \leq k \leq n-1, \alpha \geq \frac{1}{k}$ and $\mathcal{F} \geq 0$, then $M$ is a slice $\left\{r_{0}\right\} \times \mathbb{S}^{n}$ in $\mathbb{S}_{+}^{n+1}$.

Corollary 1.5. Let $M$ be a closed, strictly convex hypersurface in the hemisphere $\mathbb{S}_{+}^{n+1}$ satisfying

$$
S_{k}^{\alpha}(\kappa)-\mathcal{F}=\bar{g}\left(\lambda \partial_{r}, \nu\right) .
$$

If $k \geq 1, \alpha \geq \frac{1}{k}$ and $\mathcal{F} \geq 0$, then $M$ is a slice $\left\{r_{0}\right\} \times \mathbb{S}^{n}$ in $\mathbb{S}_{+}^{n+1}$.

For the power of Gauss curvature case, i.e., $F=\sigma_{n}^{\alpha}$, we have the following corollary.

Corollary 1.6. Let $M$ be a closed, strictly convex hypersurface in the hemisphere $\mathbb{S}_{+}^{n+1}$ satisfying

$$
\sigma_{n}^{\alpha}(\kappa)-\mathcal{F}=\bar{g}\left(\lambda \partial_{r}, \nu\right) .
$$

If $\alpha \geq \frac{1}{n+2}$ and $\mathcal{F} \geq 0$, then $M$ is a slice $\left\{r_{0}\right\} \times \mathbb{S}^{n}$ in $\mathbb{S}_{+}^{n+1}$.

Remark 1.7. In Euclidean space, $M$ is an ellipsoid under the same conditions when $\alpha=\frac{1}{n+2}$ (See $\left.[2,5]\right)$. But in the hemisphere, the positivity of the sectional curvatures of the ambient manifold forces $M$ to be umbilic.

In 3-dimensional hyperbolic space $\mathbb{H}^{3}$, deforming surfaces by a speed function $\sigma_{2}-1$ is studied in [3]. For self-similar solutions to a relevant curvature flow in $\mathbb{H}^{3}$, we obtain the following theorem.

Theorem 1.8. Let $M$ be a closed, strictly convex surface in $\mathbb{H}^{3}$ satisfying

$$
\sigma_{2}(\kappa)-\mathcal{F}=\bar{g}\left(\lambda \partial_{r}, \nu\right) .
$$

If $\mathcal{F} \geq 1$, then $M$ is a slice $\left\{r_{0}\right\} \times \mathbb{S}^{n}$ in $\mathbb{H}^{3}$.

The paper is organized as follows. In Section 2, we present basic properties of curvature tensors in warped products, then we derive some fundamental formulas for self-similar solutions in warped products with a general curvature function $F$ satisfying Condition 1.1. In Section 3 and Section 4, we use a two-step maximum principle to prove the case $\beta>1$ for Theorem 1.2. The case for $\beta=1$ is proved in Section 5. In the last section, we finish the proof of Corollary 1.6 and Theorem 1.8. Throughout this paper, the summation convention is used unless otherwise stated.

\section{Preliminaries}

Let $N$ be a warped product of the form $N=[0, \bar{r}) \times \mathbb{S}^{n}$ endowed with metric $\bar{g}=d r^{2}+\lambda^{2}(r) g_{\mathrm{s}}$. Suppose that $M^{n}(n \geq 2)$ is a smooth closed strictly convex embedded orientable hypersurface in $N$ satisfying

$$
F-\mathcal{F}=\bar{g}\left(\lambda \partial_{r}, \nu\right),
$$

described as above. Let $h=\left(h_{i j}\right)$ denote the second fundamental form with respect to an orthogonal frame $\left\{e_{1}, \ldots, e_{n}\right\}$ on $M$. The principal curvatures $\kappa_{1}, \ldots, \kappa_{n}$ are the eigenvalues of $h$. 
For convenience we first state the properties of curvature tensors of $(N, \bar{g})$. Our convention for the $(1,3)$ - and $(0,4)$-Riemannian curvature tensors of the Levi-Civita connection $\bar{\nabla}$ of $(N, \bar{g})$ are given by

$$
\bar{R}\left(Y_{1}, Y_{2}\right) Y_{3}=\bar{\nabla}_{Y_{1}} \bar{\nabla}_{Y_{2}} Y_{3}-\bar{\nabla}_{Y_{2}} \bar{\nabla}_{Y_{1}} Y_{3}-\bar{\nabla}_{\left[Y_{1}, Y_{2}\right]} Y_{3}
$$

and

$$
\bar{R}\left(Y_{1}, Y_{2}, Y_{3}, Y_{4}\right)=-\bar{g}\left(\bar{R}\left(Y_{1}, Y_{2}\right) Y_{3}, Y_{4}\right),
$$

respectively, for vector fields $Y_{1}, Y_{2}, Y_{3}, Y_{4}$ on $N$. Thus the $(0,4)$-Riemannian curvature tensor of $(N, \bar{g})$ is

$$
\bar{R}=\frac{1-\lambda^{\prime 2}}{2 \lambda^{2}} \bar{g} \otimes \bar{g}-\left(\frac{1-\lambda^{\prime 2}}{\lambda^{2}}+\frac{\lambda^{\prime \prime}}{\lambda}\right) \bar{g} \otimes d r^{2},
$$

where (A) is the Kulkarni-Nomizu product, cf. [13].

In terms of orthonormal frames $\left\{e_{1}, \cdots, e_{n}, \nu\right\}$ of $N$ along $M$, we use the conventions $\bar{R}_{i j k l}=\bar{R}\left(e_{i}, e_{j}, e_{k}, e_{l}\right)$ and $\bar{R}_{\nu i j k}=\bar{R}\left(\nu, e_{i}, e_{j}, e_{k}\right)$. Denote $r_{i}=\bar{g}\left(\partial_{r}, e_{i}\right)$ and $r_{\nu}=\bar{g}\left(\partial_{r}, \nu\right)$. We have

$\bar{R}_{i j k l}=\frac{1-\lambda^{\prime 2}}{\lambda^{2}}\left(\delta_{i k} \delta_{j l}-\delta_{i l} \delta_{j k}\right)-\left(\frac{1-\lambda^{\prime 2}}{\lambda^{2}}+\frac{\lambda^{\prime \prime}}{\lambda}\right)\left(\delta_{i k} r_{j} r_{l}+\delta_{j l} r_{i} r_{k}-\delta_{i l} r_{j} r_{k}-\delta_{j k} r_{i} r_{l}\right)$,

and

$$
\bar{R}_{\nu i j k}=-\left(\frac{1-\lambda^{\prime 2}}{\lambda^{2}}+\frac{\lambda^{\prime \prime}}{\lambda}\right) r_{\nu}\left(\delta_{i k} r_{j}-\delta_{i j} r_{k}\right)
$$

Let $\nabla$ denote the Levi-Civita connection with respect to the induced metric on $M$. It follows from a direct computation that the covariant derivatives of $r_{\nu}$ and $r_{k}$ are given by

$$
r_{\nu ; l}=\nabla_{l} r_{\nu}=\nabla_{l} \bar{g}\left(\partial_{r}, \nu\right)=-\frac{\lambda^{\prime}}{\lambda} r_{l} r_{\nu}+h_{l m} r_{m}
$$

and

$$
r_{k ; l}=\nabla_{l} r_{k}=\frac{\lambda^{\prime}}{\lambda}\left(\delta_{k l}-r_{k} r_{l}\right)-h_{k l} r_{\nu}
$$

Thus we obtain the following covariant derivative of the curvature tensor

$$
\begin{aligned}
\bar{R}_{\nu i j k ; l} & =\left\{-\left(\frac{1-\lambda^{\prime 2}}{\lambda^{2}}+\frac{\lambda^{\prime \prime}}{\lambda}\right)^{\prime}+2 \frac{\lambda^{\prime}}{\lambda}\left(\frac{1-\lambda^{\prime 2}}{\lambda^{2}}+\frac{\lambda^{\prime \prime}}{\lambda}\right)\right\} r_{l} r_{\nu}\left(\delta_{i k} r_{j}-\delta_{i j} r_{k}\right) \\
& +\left(\frac{1-\lambda^{\prime 2}}{\lambda^{2}}+\frac{\lambda^{\prime \prime}}{\lambda}\right)\left\{-h_{l m} r_{m}\left(\delta_{i k} r_{j}-\delta_{i j} r_{k}\right)-\frac{\lambda^{\prime}}{\lambda} r_{\nu}\left(\delta_{i k} \delta_{j l}-\delta_{i j} \delta_{k l}\right)+r_{\nu}^{2}\left(\delta_{i k} h_{j l}-\delta_{i j} h_{k l}\right)\right\} .
\end{aligned}
$$

Denote $h_{i j k}=\nabla_{k} h_{i j}$ and $h_{i j k l}=\nabla_{l} \nabla_{k} h_{i j}$. Making use of the Gauss equation

$$
R_{i j k l}=\bar{R}_{i j k l}+h_{i k} h_{j l}-h_{i l} h_{j k},
$$

the Coddazi equation

$$
h_{i j k}=h_{i k j}+\bar{R}_{\nu i j k}
$$

and the Ricci identity, we get

$$
\begin{aligned}
h_{i j k l} & =h_{i k j l}+\bar{R}_{\nu i j k ; l} \\
& =h_{k i l j}+h_{m k} R_{m i j l}+h_{i m} R_{m k j l}+\bar{R}_{\nu i j k ; l} \\
& =h_{k l i j}+h_{m k}\left(h_{m j} h_{i l}-h_{i j} h_{m l}\right)+h_{i m}\left(h_{m j} h_{k l}-h_{m l} h_{j k}\right) \\
& +\bar{R}_{\nu k i l ; j}+\bar{R}_{\nu i j k ; l}+h_{m k} \bar{R}_{m i j l}+h_{i m} \bar{R}_{m k j l} .
\end{aligned}
$$


By straightforward calculation, we have

$$
\begin{aligned}
& \bar{R}_{\nu k i l ; j}+\bar{R}_{\nu i j k ; l}+h_{m k} \bar{R}_{m i j l}+h_{i m} \bar{R}_{m k j l} \\
= & \left\{-\left(\frac{1-\lambda^{\prime 2}}{\lambda^{2}}+\frac{\lambda^{\prime \prime}}{\lambda}\right)^{\prime}+2 \frac{\lambda^{\prime}}{\lambda}\left(\frac{1-\lambda^{\prime 2}}{\lambda^{2}}+\frac{\lambda^{\prime \prime}}{\lambda}\right)\right\} r_{\nu}\left(\delta_{k l} r_{i} r_{j}-\delta_{i j} r_{k} r_{l}\right) \\
& +\frac{1-\lambda^{\prime 2}}{\lambda^{2}}\left(h_{k j} \delta_{i l}-h_{k l} \delta_{i j}+h_{i j} \delta_{k l}-h_{i l} \delta_{k j}\right) \\
& -\left(\frac{1-\lambda^{\prime 2}}{\lambda^{2}}+\frac{\lambda^{\prime \prime}}{\lambda}\right)\left(h_{j k} r_{i} r_{l}-h_{k l} r_{i} r_{j}+h_{i j} r_{k} r_{l}-h_{i l} r_{k} r_{j}\right) \\
& -\left(\frac{1-\lambda^{\prime 2}}{\lambda^{2}}+\frac{\lambda^{\prime \prime}}{\lambda}\right) r_{m}\left(\delta_{k l} r_{i} h_{j m}-\delta_{k i} r_{l} h_{j m}+\delta_{i k} r_{j} h_{l m}-\delta_{i j} r_{k} h_{l m}\right. \\
& \left.+h_{m k} \delta_{i l} r_{j}-h_{m k} \delta_{i j} r_{l}+h_{i m} \delta_{k l} r_{j}-h_{i m} \delta_{k j} r_{l}\right) .
\end{aligned}
$$

Let $b=\left(b^{i j}\right)$ denote the inverse of the second fundamental form $h=\left(h_{i j}\right)$ with respect to a given orthonormal frame $\left\{e_{1}, \cdots, e_{n}\right\}$ of $M$. Define the operator $\mathcal{L}$ by $\mathcal{L}=\frac{\partial F}{\partial h_{i j}} \nabla_{i} \nabla_{j}$. It follows from Condition 1.1 that $\mathcal{L}$ is an elliptic operator. Define a function $Z$ by

$$
Z=F \operatorname{tr} b-\frac{n(\beta-1)}{\beta} \Phi,
$$

where $\Phi=\int_{0}^{r} \lambda(s) d s$. We next derive some basic formulas of $\mathcal{L}$ for further use.

Proposition 2.1. Given a smooth function $F: M \rightarrow \mathbb{R}$ described as above, the following equations hold:

$$
\begin{aligned}
(1) \mathcal{L} F= & \bar{g}\left(\lambda \partial_{r}, \nabla F\right)+\beta \lambda^{\prime} F-\frac{\partial F}{\partial h_{i j}} h_{i l} h_{j l}(F-\mathcal{F})+\frac{\partial F}{\partial h_{i j}} \bar{R}_{\nu j l i} \bar{g}\left(\lambda \partial_{r}, e_{l}\right), \\
(2) \quad \mathcal{L} h_{k l}= & \bar{g}\left(\lambda \partial_{r}, \nabla h_{l k}\right)+\lambda^{\prime} h_{l k}+h_{l m} h_{k m} \mathcal{F}+\bar{R}_{\nu k m l} \bar{g}\left(\lambda \partial_{r}, e_{m}\right) \\
& -\frac{\partial^{2} F}{\partial h_{i j} \partial h_{s t}} h_{i j k} h_{s t l}-\frac{\partial F}{\partial h_{i j}} h_{m j} h_{m i} h_{k l}+(\beta-1) F h_{k m} h_{m l} \\
& +\frac{\partial F}{\partial h_{i j}}\left(\bar{R}_{\nu i k j ; l}+\bar{R}_{\nu k l i j j}+h_{m i} \bar{R}_{m k l j}+h_{k m} \bar{R}_{m i l j}\right), \\
(3) \quad \mathcal{L} b^{k l}= & \bar{g}\left(\lambda \partial_{r}, \nabla b^{k l}\right)-\lambda^{\prime} b^{k l}-\delta_{k l} \mathcal{F}-b^{k p} b^{q l} \bar{R}_{\nu p m q} \bar{g}\left(\lambda \partial_{r}, e_{m}\right) \\
& +b^{k p} b^{q l} \frac{\partial^{2} F}{\partial h_{i j} \partial h_{s t}} h_{i j p} h_{s t q}+\frac{\partial F}{\partial h_{i j}} h_{m j} h_{m i} b^{k l}-(\beta-1) F \delta_{k l} \\
& -b^{k p} b^{q l} \frac{\partial F}{\partial h_{i j}}\left(\bar{R}_{\nu i p j ; q}+\bar{R}_{\nu p q i ; j}+h_{m i} \bar{R}_{m p q j}+h_{p m} \bar{R}_{m i q j}\right) \\
& +2 b^{k s} b^{p t} b^{l q} \frac{\partial F}{\partial h_{i j}} h_{s t i} h_{p q j}, \\
\mathcal{L} \Phi= & \lambda^{\prime} \sum_{i} \frac{\partial F}{\partial h_{i i}}-\beta F(F-\mathcal{F}), \\
\mathcal{L} Z= & 2 \frac{\partial F}{\partial h_{i j}} \nabla_{i} F \nabla_{j} \operatorname{tr} b+\bar{g}\left(\lambda \partial_{r}, \nabla(F \operatorname{tr} b)\right)+(\beta-1) \lambda^{\prime}\left(F \operatorname{tr} b-\frac{n}{\beta} \sum_{i} \frac{\partial F}{\partial h_{i i}}\right) \\
& +\left(\frac{\partial F}{\partial h_{i j}} h_{i l} h_{j l} \operatorname{tr} b-\beta n F\right) \mathcal{F}+F b^{k p} b^{q k} \frac{\partial^{2} F}{\partial h_{i j} \partial h_{s t}} h_{i j p} h_{s t q} \\
(5) \quad &
\end{aligned}
$$




$$
\begin{aligned}
& +2 F b^{k s} b^{p t} b^{k q} \frac{\partial F}{\partial h_{i j}} h_{s t i} h_{p q j}+\left(\operatorname{tr} b \frac{\partial F}{\partial h_{i j}}-F b^{k i} b^{j k}\right) \bar{R}_{\nu i m j} \bar{g}\left(\lambda \partial_{r}, e_{m}\right) \\
& -F b^{k p} b^{q k} \frac{\partial F}{\partial h_{i j}}\left(\bar{R}_{\nu i p j ; q}+\bar{R}_{\nu p q i ; j}+h_{m i} \bar{R}_{m p q j}+h_{p m} \bar{R}_{m i q j}\right) .
\end{aligned}
$$

Proof. (1) From

$$
\bar{\nabla}_{e_{i}} \lambda \partial_{r}=\lambda^{\prime} e_{i}
$$

we know

$$
\nabla_{i} F=\bar{g}\left(\lambda \partial_{r}, h_{i l} e_{l}\right)
$$

and

$$
\begin{aligned}
\nabla_{i} \nabla_{j} F & =h_{j l i} \bar{g}\left(\lambda \partial_{r}, e_{l}\right)+\lambda^{\prime} h_{i j}-h_{i l} h_{j l}(F-\mathcal{F}) \\
& =h_{i j l} \bar{g}\left(\lambda \partial_{r}, e_{l}\right)+\lambda^{\prime} h_{i j}-h_{i l} h_{j l}(F-\mathcal{F})+\bar{R}_{\nu j l i} \bar{g}\left(\lambda \partial_{r}, e_{l}\right) .
\end{aligned}
$$

Then from $\frac{\partial F}{\partial h_{i j}} h_{i j}=\beta F$ we get

$$
\mathcal{L} F=\bar{g}\left(\lambda \partial_{r}, \nabla F\right)+\beta \lambda^{\prime} F-\frac{\partial F}{\partial h_{i j}} h_{i l} h_{j l}(F-\mathcal{F})+\frac{\partial F}{\partial h_{i j}} \bar{R}_{\nu j l i} \bar{g}\left(\lambda \partial_{r}, e_{l}\right) .
$$

(2) From (2.4), we have

$$
\begin{aligned}
& \mathcal{L} h_{k l}=\frac{\partial F}{\partial h_{i j}} h_{k l i j} \\
= & \frac{\partial F}{\partial h_{i j}}\left(h_{i j k l}+h_{m i}\left(h_{m l} h_{k j}-h_{k l} h_{m j}\right)+h_{k m}\left(h_{m l} h_{i j}-h_{m j} h_{l i}\right)\right. \\
+ & \left.\bar{R}_{\nu i k j ; l}+\bar{R}_{\nu k l i ; j}+h_{m i} \bar{R}_{m k l j}+h_{k m} \bar{R}_{m i l j}\right) \\
= & \nabla_{l} \nabla_{k} F-\frac{\partial^{2} F}{\partial h_{i j} \partial h_{s t}} h_{i j k} h_{s t l}-\frac{\partial F}{\partial h_{i j}} h_{m j} h_{m i} h_{k l}+\beta F h_{k m} h_{m l} \\
+ & \frac{\partial F}{\partial h_{i j}}\left(\bar{R}_{\nu i k j ; l}+\bar{R}_{\nu k l i ; j}+h_{m i} \bar{R}_{m k l j}+h_{k m} \bar{R}_{m i l j}\right) \\
= & \bar{g}\left(\lambda \partial_{r}, \nabla h_{l k}\right)+\lambda^{\prime} h_{l k}+h_{l m} h_{k m} \mathcal{F}+\bar{R}_{\nu k m l} \bar{g}\left(\lambda \partial_{r}, e_{m}\right) \\
& -\frac{\partial^{2} F}{\partial h_{i j} \partial h_{s t}} h_{i j k} h_{s t l}-\frac{\partial F}{\partial h_{i j}} h_{m j} h_{m i} h_{k l}+(\beta-1) F h_{k m} h_{m l} \\
& +\frac{\partial F}{\partial h_{i j}}\left(\bar{R}_{\nu i k j ; l}+\bar{R}_{\nu k l i ; j}+h_{m i} \bar{R}_{m k l j}+h_{k m} \bar{R}_{m i l j}\right) .
\end{aligned}
$$

(3) Since $h_{k m} b^{m l}=\delta_{k l}$, we have

$$
\nabla_{j} b^{k l}=-b^{k p} b^{l q} \nabla_{j} h_{p q} .
$$

And,

$$
\begin{aligned}
\nabla_{i} \nabla_{j} b^{k l} & =-\nabla_{i}\left(b^{k p} b^{l q} \nabla_{j} h_{p q}\right) \\
& =-b^{k p} b^{q l} \nabla_{i} \nabla_{j} h_{p q}+b^{k s} b^{p t} b^{l q} \nabla_{i} h_{s t} \nabla_{j} h_{p q}+b^{k p} b^{l s} b^{q t} \nabla_{i} h_{s t} \nabla_{j} h_{p q} .
\end{aligned}
$$


Then, using (2) we obtain

$$
\begin{aligned}
\mathcal{L} b^{k l} & =-b^{k p} b^{q l} \frac{\partial F}{\partial h_{i j}} \nabla_{i} \nabla_{j} h_{p q}+2 b^{k s} b^{p t} b^{l q} \frac{\partial F}{\partial h_{i j}} \nabla_{i} h_{s t} \nabla_{j} h_{p q} \\
& =-b^{k p} b^{q l}\left(\bar{g}\left(\lambda \partial_{r}, \nabla h_{p q}\right)+\lambda^{\prime} h_{p q}+h_{p m} h_{q m} \mathcal{F}+\bar{R}_{\nu p m q} \bar{g}\left(\lambda \partial_{r}, e_{m}\right)\right. \\
& -\frac{\partial^{2} F}{\partial h_{i j} \partial h_{s t}} h_{i j p} h_{s t q}-\frac{\partial F}{\partial h_{i j}} h_{m j} h_{m i} h_{p q}+(\beta-1) F h_{p m} h_{m q} \\
& \left.+\frac{\partial F}{\partial h_{i j}}\left(\bar{R}_{\nu i p j ; q}+\bar{R}_{\nu p q i j}+h_{m i} \bar{R}_{m p q j}+h_{p m} \bar{R}_{m i q j}\right)\right) \\
& +2 b^{k s} b^{p t} b^{l q} \frac{\partial F}{\partial h_{i j}} h_{s t i} h_{p q j} \\
& =\bar{g}\left(\lambda \partial_{r}, \nabla b^{k l}\right)-\lambda^{\prime} b^{k l}-\delta_{k l} \mathcal{F}-b^{k p} b^{q l} \bar{R}_{\nu p m q} \bar{g}\left(\lambda \partial_{r}, e_{m}\right) \\
& +b^{k p} b^{q l} \frac{\partial^{2} F}{\partial h_{i j} \partial h_{s t}} h_{i j p} h_{s t q}+\frac{\partial F}{\partial h_{i j}} h_{m j} h_{m i} b^{k l}-(\beta-1) F \delta_{k l} \\
& -b^{k p} b^{q l} \frac{\partial F}{\partial h_{i j}}\left(\bar{R}_{\nu i p j ; q}+\bar{R}_{\nu p q i j}+h_{m i} \bar{R}_{m p q j}+h_{p m} \bar{R}_{m i q j}\right) \\
& +2 b^{k s} b^{p t} b^{l q} \frac{\partial F}{\partial h_{i j}} h_{s t i} h_{p q j} .
\end{aligned}
$$

(4) We know

$$
\nabla_{i} \Phi=\bar{\nabla}_{e_{i}} \Phi=\lambda(r) \bar{\nabla}_{e_{i}} r=\bar{g}\left(\lambda \partial_{r}, e_{i}\right)
$$

and

$$
\nabla_{i} \nabla_{j} \Phi=\lambda^{\prime} \delta_{i j}-h_{i j} \bar{g}\left(\lambda \partial_{r}, \nu\right)=\lambda^{\prime} \delta_{i j}-h_{i j}(F-\mathcal{F})
$$

Then

$$
\mathcal{L} \Phi=\lambda^{\prime} \sum_{i} \frac{\partial F}{\partial h_{i i}}-\beta F(F-\mathcal{F})
$$

(5) From (4) we know

$$
\begin{aligned}
\mathcal{L} \operatorname{tr} b & =\bar{g}\left(\lambda \partial_{r}, \nabla \operatorname{tr} b\right)-\lambda^{\prime} \operatorname{tr} b-n \mathcal{F}-b^{k p} b^{q k} \bar{R}_{\nu p m q} \bar{g}\left(\lambda \partial_{r}, e_{m}\right) \\
& +b^{k p} b^{q k} \frac{\partial^{2} F}{\partial h_{i j} \partial h_{s t}} h_{i j p} h_{s t q}+\frac{\partial F}{\partial h_{i j}} h_{m j} h_{m i} \operatorname{tr} b-n(\beta-1) F \\
& -b^{k p} b^{q k} \frac{\partial F}{\partial h_{i j}}\left(\bar{R}_{\nu i p j ; q}+\bar{R}_{\nu p q i ; j}+h_{m i} \bar{R}_{m p q j}+h_{p m} \bar{R}_{m i q j}\right) \\
& +2 b^{k s} b^{p t} b^{k q} \frac{\partial F}{\partial h_{i j}} h_{s t i} h_{p q j} .
\end{aligned}
$$


Then we have

$$
\begin{aligned}
\mathcal{L} Z & =2 \frac{\partial F}{\partial h_{i j}} \nabla_{i} F \nabla_{j} \operatorname{tr} b+\operatorname{tr} b \mathcal{L} F+F \mathcal{L} \operatorname{tr} b-\frac{n(\beta-1)}{\beta} \mathcal{L} \Phi \\
& =2 \frac{\partial F}{\partial h_{i j}} \nabla_{i} F \nabla_{j} \operatorname{tr} b+\operatorname{tr} b \bar{g}\left(\lambda \partial_{r}, \nabla F\right)+\beta \lambda^{\prime} F \operatorname{tr} b \\
& -\frac{\partial F}{\partial h_{i j}} h_{i l} h_{j l}(F-\mathcal{F}) \operatorname{tr} b+\operatorname{tr} b \frac{\partial F}{\partial h_{i j}} \bar{R}_{\nu j l i} \bar{g}\left(\lambda \partial_{r}, e_{l}\right) \\
& +F \bar{g}\left(\lambda \partial_{r}, \nabla \operatorname{tr} b\right)-\lambda^{\prime} F \operatorname{tr} b-n F \mathcal{F}-F b^{k p} b^{q k} \bar{R}_{\nu p m q} \bar{g}\left(\lambda \partial_{r}, e_{m}\right) \\
& +F b^{k p} b^{q k} \frac{\partial^{2} F}{\partial h_{i j} \partial h_{s t}} h_{i j p} h_{s t q}+F \frac{\partial F}{\partial h_{i j}} h_{m j} h_{m i} \operatorname{tr} b-n(\beta-1) F^{2} \\
& -F b^{k p} b^{q k} \frac{\partial F}{\partial h_{i j}}\left(\bar{R}_{\nu i p j ; q}+\bar{R}_{\nu p q i j}+h_{m i} \bar{R}_{m p q j}+h_{p m} \bar{R}_{m i q j}\right) \\
& +2 F b^{k s} b^{p t} b^{k q} \frac{\partial F}{\partial h_{i j}} h_{s t i} h_{p q j}-\frac{n(\beta-1)}{\beta} \lambda^{\prime} \sum_{i} \frac{\partial F}{\partial h_{i i}}+n(\beta-1) F(F-\mathcal{F}) \\
& =2 \frac{\partial F}{\partial h_{i j}} \nabla_{i} F \nabla_{j} \operatorname{tr} b+\bar{g}\left(\lambda \partial_{r}, \nabla(F \operatorname{tr} b)\right)+(\beta-1) \lambda^{\prime}\left(F \operatorname{tr} b-\frac{n}{\beta} \sum_{i} \frac{\partial F}{\partial h_{i i}}\right) \\
& +\left(\frac{\partial F}{\partial h_{i j}} h_{i l} h_{j l} \operatorname{tr} b-\beta n F\right) \mathcal{F}+F b^{k p} b^{q k} \frac{\partial^{2} F}{\partial h_{i j} \partial h_{s t}} h_{i j p} h_{s t q} \\
& +2 F b^{k s} b^{p t} b^{k q} \frac{\partial F}{\partial h_{i j}} h_{s t i} h_{p q j}+\left(\operatorname{tr} b \frac{\partial F}{\partial h_{i j}}-F b^{k i} b^{j k}\right) \bar{R}_{\nu i m j} \bar{g}\left(\lambda \partial_{r}, e_{m}\right) \\
& -F b^{k p} b^{q k} \frac{\partial F}{\partial h_{i j}}\left(\bar{R}_{\nu i p j ; q}+\bar{R}_{\nu p q i ; j}+h_{m i} \bar{R}_{m p q j}+h_{p m} \bar{R}_{m i q j}\right) .
\end{aligned}
$$

Notice that for a warped product $N$, when $\lambda(r)=r, \sin r$, or $\sinh r, N$ is Euclidean space, the sphere $\mathbb{S}^{n+1}$ or hyperbolic space $\mathbb{H}^{n+1}$ with constant sectional curvature $\epsilon=0,1$ or -1 respectively. For the rest of the paper, we focus on spaces of constant sectional curvature. In these cases, we have

$$
\bar{R}_{i j k l}=\epsilon\left(\delta_{i k} \delta_{j l}-\delta_{i l} \delta_{j k}\right) \text { and } \bar{R}_{\nu i j k}=0 .
$$

Therefore,

$$
\begin{aligned}
& \bar{R}_{\nu k m l} \bar{g}\left(\lambda \partial_{r}, e_{m}\right)+\frac{\partial F}{\partial h_{i j}}\left(\bar{R}_{\nu i k j ; l}+\bar{R}_{\nu k l i ; j}+h_{m i} \bar{R}_{m k l j}+h_{k m} \bar{R}_{m i l j}\right) \\
= & \epsilon \frac{\partial F}{\partial h_{i j}}\left(h_{m i}\left(\delta_{m l} \delta_{k j}-\delta_{m j} \delta_{k l}\right)+h_{k m}\left(\delta_{m l} \delta_{i j}-\delta_{m j} \delta_{i l}\right)\right) \\
= & \epsilon \frac{\partial F}{\partial h_{i j}}\left(h_{i l} \delta_{k j}-h_{i j} \delta_{k l}+h_{k l} \delta_{i j}-h_{k j} \delta_{i l}\right)
\end{aligned}
$$


and

$$
\begin{aligned}
& \left(\operatorname{tr} b \frac{\partial F}{\partial h_{i j}}-F b^{k i} b^{j k}\right) \bar{R}_{\nu i m j} \bar{g}\left(\lambda \partial_{r}, e_{m}\right) \\
- & F b^{k p} b^{q k} \frac{\partial F}{\partial h_{i j}}\left(\bar{R}_{\nu i p j ; q}+\bar{R}_{\nu p q i ; j}+h_{m i} \bar{R}_{m p q j}+h_{p m} \bar{R}_{m i q j}\right) \\
= & -\epsilon F b^{k p} b^{q k} \frac{\partial F}{\partial h_{i j}}\left(h_{i q} \delta_{p j}-h_{p j} \delta_{i q}+h_{p q} \delta_{i j}-h_{i j} \delta_{p q}\right) \\
= & \epsilon F\left(\beta F \operatorname{tr}\left(b^{2}\right)-\operatorname{tr} b \sum_{i} \frac{\partial F}{\partial h_{i i}}\right)
\end{aligned}
$$

Then in spaces of constant sectional curvature, (2) and (4) in Proposition 2.1 reduce the following equations.

\section{Corollary 2.2.}

$$
\begin{aligned}
\mathcal{L} h_{k l} & =\bar{g}\left(\lambda \partial_{r}, \nabla h_{l k}\right)+\lambda^{\prime} h_{l k}+h_{l m} h_{k m} \mathcal{F}-\frac{\partial^{2} F}{\partial h_{i j} \partial h_{s t}} h_{i j k} h_{s t l}-\frac{\partial F}{\partial h_{i j}} h_{m j} h_{m i} h_{k l} \\
& +(\beta-1) F h_{k m} h_{m l}+\epsilon \frac{\partial F}{\partial h_{i j}}\left(h_{i l} \delta_{k j}-h_{i j} \delta_{k l}+h_{k l} \delta_{i j}-h_{k j} \delta_{i l}\right), \\
\mathcal{L} Z & =2 \frac{\partial F}{\partial h_{i j}} \nabla_{i} F \nabla_{j} \operatorname{tr} b+\bar{g}\left(\lambda \partial_{r}, \nabla(F \operatorname{tr} b)\right)+(\beta-1) \lambda^{\prime}\left(F \operatorname{tr} b-\frac{n}{\beta} \sum_{i} \frac{\partial F}{\partial h_{i i}}\right) \\
& +\left(\frac{\partial F}{\partial h_{i j}} h_{i l} h_{j l} \operatorname{tr} b-\beta n F\right) \mathcal{F}+F b^{k p} b^{q k} \frac{\partial^{2} F}{\partial h_{i j} \partial h_{s t}} h_{i j p} h_{s t q} \\
& +2 F b^{k s} b^{p t} b^{k q} \frac{\partial F}{\partial h_{i j}} h_{s t i} h_{p q j}+\epsilon F\left(\beta F \operatorname{tr}\left(b^{2}\right)-\operatorname{tr} b \sum_{i} \frac{\partial F}{\partial h_{i i}}\right) .
\end{aligned}
$$

For convenience, we call the following term

$$
\epsilon F\left(\beta F \operatorname{tr}\left(b^{2}\right)-\operatorname{tr} b \sum_{i} \frac{\partial F}{\partial h_{i i}}\right)
$$

the $\epsilon$-term in $\mathcal{L} Z$. It vanishes for the Euclidean space case and we need to estimate it in other cases. In fact, we have the following lemma.

Lemma 2.3. If $F$ satisfies $i)$, ii) and iii) in Condition 1.1 and $\kappa \in \Gamma_{+}$, we have

$$
\beta F \operatorname{tr}\left(b^{2}\right)-\operatorname{tr} b \sum_{i} \frac{\partial F}{\partial h_{i i}} \geq 0
$$

and the equality occurs if and only if $\kappa_{1}=\ldots=\kappa_{n}$.

Proof. In fact,

$$
\begin{aligned}
& \beta F \operatorname{tr}\left(b^{2}\right)-\operatorname{tr} b \sum_{i} \frac{\partial F}{\partial h_{i i}}=\sum_{i, j} \frac{\partial F}{\partial \kappa_{i}}\left(\kappa_{i} \kappa_{j}^{-2}-\kappa_{j}^{-1}\right) \\
= & \sum_{i \neq j} \kappa_{i}^{-2} \kappa_{j}^{-2} \frac{\partial F}{\partial \kappa_{i}} \kappa_{i}^{2}\left(\kappa_{i}-\kappa_{j}\right)=\sum_{i>j} \kappa_{i}^{-2} \kappa_{j}^{-2}\left(\frac{\partial F}{\partial \kappa_{i}} \kappa_{i}^{2}-\frac{\partial F}{\partial \kappa_{i}} \kappa_{i}^{2}\right)\left(\kappa_{i}-\kappa_{j}\right) .
\end{aligned}
$$

Using i), ii) and iii) in Condition 1.1, we finish the proof. 


\section{AnAlysis at the maXimum points of $W$}

In this section and next section, we prove Theorem 1.2 for $\beta>1$. The proof is a delicate application of the maximum principle to two test functions $W=\frac{F}{\kappa_{1}}-\frac{\beta-1}{\beta} \Phi$ and $Z$, where $\kappa_{1}$ is the smallest principal curvature of $M$. The idea comes from $[6,5]$ and is used in [9]. The following lemma is employed to analyze the maximum points of $W$.

Lemma 3.1 ([5]). Let $\mu$ denote the multiplicity of $\kappa_{1}$ at a point $\bar{x}$, i.e., $\kappa_{1}(\bar{x})=$ $\cdots=\kappa_{\mu}(\bar{x})<\kappa_{\mu+1}(\bar{x})$. Suppose that $\varphi$ is a smooth function such that $\varphi \leq \kappa_{1}$ everywhere and $\varphi(\bar{x})=\kappa_{1}(\bar{x})$. Then, at $\bar{x}$, we have

i) $h_{k l i}=\nabla_{i} \varphi \delta_{k l}$ for $1 \leq k, l \leq \mu$.

ii) $\nabla_{i} \nabla_{i} \varphi \leq h_{11 i i}-2 \sum_{l>\mu}\left(\kappa_{l}-\kappa_{1}\right)^{-1} h_{1 l i}^{2}$.

Now define a smooth function $\varphi$ by $\frac{F}{\varphi}-\frac{\beta-1}{\beta} \Phi=\max _{x \in M} W(x)$ on $M$. If $W$ attains its maximum at $\bar{x}$, then we know $\varphi \leq \kappa_{1}$ everywhere and $\varphi(\bar{x})=\kappa_{1}(\bar{x})$.

Using Lemma 3.1 at $\bar{x}$ and $(i)$ in Corollary 2.2, we have

$$
\begin{aligned}
\mathcal{L} \varphi & \leq \mathcal{L} h_{11}-2 \frac{\partial F}{\partial \kappa_{i}} \sum_{l>\mu}\left(\kappa_{l}-\kappa_{1}\right)^{-1} h_{1 l i}^{2} \\
& =\bar{g}\left(\lambda \partial_{r}, \nabla h_{11}\right)+\lambda^{\prime} \kappa_{1}+\mathcal{F} \kappa_{1}^{2}-\kappa_{1} \frac{\partial F}{\partial h_{i j}} h_{m j} h_{m i}+\kappa_{1}^{2}(\beta-1) F-\frac{\partial^{2} F}{\partial h_{i j} \partial h_{s t}} h_{i j 1} h_{s t 1} \\
& -2 \frac{\partial F}{\partial \kappa_{i}} \sum_{l>\mu}\left(\kappa_{l}-\kappa_{1}\right)^{-1} h_{1 l i}^{2}-\epsilon \beta F+\epsilon \kappa_{1} \sum_{i} \frac{\partial F}{\partial h_{i i}} .
\end{aligned}
$$

Lemma 3.2. Let $M$ be a strictly convex hypersurface in the hemisphere $\mathbb{S}_{+}^{n+1}$ with $n \geq 2$ satisfying (1.1). For $\beta \geq 1$ and $\mathcal{F} \geq 0$, if $F$ satisfies Condition 1.1 and $\bar{x}$ is a maximum point of $W$, then $\bar{x}$ must be umbilic and $\nabla F(\bar{x})=0$.

Proof. At $\bar{x}$, we have

$$
0=\nabla_{i}\left(\frac{F}{\varphi}-\frac{\beta-1}{\beta} \Phi\right)
$$

for $1 \leq i \leq n$. And, using (3.1), we obtain

$$
\begin{aligned}
0 & =\mathcal{L}\left(\frac{F}{\varphi}-\frac{\beta-1}{\beta} \Phi\right) \\
\geq & \bar{g}\left(\lambda \partial_{r}, \nabla\left(\frac{F}{\varphi}\right)\right)+2 \frac{\partial F}{\partial h_{i j}} \nabla_{i} F \nabla_{j} \frac{1}{\varphi}+2 F \kappa_{1}^{-3} \frac{\partial F}{\partial \kappa_{i}} h_{11 i}^{2} \\
& +F \kappa_{1}^{-2} \frac{\partial^{2} F}{\partial h_{i j} \partial h_{s t}} h_{i j 1} h_{s t 1}+2 F \kappa_{1}^{-2} \frac{\partial F}{\partial \kappa_{i}} \sum_{l>\mu}\left(\kappa_{l}-\kappa_{1}\right)^{-1} h_{1 l i}^{2} \\
& +\frac{\beta-1}{\beta} \lambda^{\prime} \frac{\partial F}{\partial \kappa_{i}}\left(\frac{\kappa_{i}}{\kappa_{1}}-1\right)+\mathcal{F} \frac{\partial F}{\partial \kappa_{i}} \kappa_{i}\left(\frac{\kappa_{i}}{\kappa_{1}}-1\right)+\epsilon F \kappa_{1}^{-1} \frac{\partial F}{\partial \kappa_{i}}\left(\frac{\kappa_{i}}{\kappa_{1}}-1\right) .
\end{aligned}
$$

For convenience, let us denote

$$
\begin{gathered}
J_{1}=\frac{\beta-1}{\beta} \lambda^{\prime} \frac{\partial F}{\partial \kappa_{i}}\left(\frac{\kappa_{i}}{\kappa_{1}}-1\right)+\mathcal{F} \frac{\partial F}{\partial \kappa_{i}} \kappa_{i}\left(\frac{\kappa_{i}}{\kappa_{1}}-1\right)+\epsilon F \kappa_{1}^{-1} \frac{\partial F}{\partial \kappa_{i}}\left(\frac{\kappa_{i}}{\kappa_{1}}-1\right) \\
J_{2}=\bar{g}\left(\lambda \partial_{r}, \nabla\left(\frac{F}{\varphi}\right)\right)+2 \frac{\partial F}{\partial h_{i j}} \nabla_{i} F \nabla_{j} \frac{1}{\varphi}+2 F \kappa_{1}^{-3} \frac{\partial F}{\partial \kappa_{i}} h_{11 i}^{2}
\end{gathered}
$$


and

$$
J_{3}=F \kappa_{1}^{-2} \frac{\partial^{2} F}{\partial h_{i j} \partial h_{s t}} h_{i j 1} h_{s t 1}+2 F \kappa_{1}^{-2} \frac{\partial F}{\partial \kappa_{i}} \sum_{l>\mu}\left(\kappa_{l}-\kappa_{1}\right)^{-1} h_{1 l i}^{2} .
$$

Using $\nabla_{i} F=\kappa_{i} \bar{g}\left(\lambda \partial_{r}, e_{i}\right), \nabla_{i} \Phi=\bar{g}\left(\lambda \partial_{r}, e_{i}\right)$ and (3.2), we have

$$
\begin{aligned}
& \bar{g}\left(\lambda \partial_{r}, \nabla\left(\frac{F}{\varphi}\right)\right)+2 \frac{\partial F}{\partial h_{i j}} \nabla_{i} F \nabla_{j} \frac{1}{\varphi} \\
= & \frac{\beta-1}{\beta} \bar{g}\left(\lambda \partial_{r}, \nabla \Phi\right)+2 F^{-1} \frac{\partial F}{\partial h_{i j}} \nabla_{i} F \nabla_{j} \frac{F}{\varphi}-\frac{2}{\varphi} F^{-1} \frac{\partial F}{\partial h_{i j}} \nabla_{i} F \nabla_{j} F \\
= & \frac{\beta-1}{\beta} \kappa_{i}^{-2}\left(\nabla_{i} F\right)^{2}+\frac{2(\beta-1)}{\beta} \kappa_{i}^{-1} \frac{\partial \log F}{\partial \kappa_{i}}\left(\nabla_{i} F\right)^{2}-2 \kappa_{1}^{-1} \frac{\partial \log F}{\partial \kappa_{i}}\left(\nabla_{i} F\right)^{2} .
\end{aligned}
$$

From (3.2) we know

$$
\kappa_{1}^{-1} \nabla_{i} F-F \kappa_{1}^{-2} h_{11 i}=\frac{\beta-1}{\beta} \nabla_{i} \Phi=\frac{\beta-1}{\beta} \kappa_{i}^{-1} \nabla_{i} F .
$$

Therefore

$$
2 F \kappa_{1}^{-3} \frac{\partial F}{\partial \kappa_{i}} h_{11 i}^{2}=2 \kappa_{1}\left(\kappa_{1}^{-1}-\frac{\beta-1}{\beta} \kappa_{i}^{-1}\right)^{2} \frac{\partial \log F}{\partial \kappa_{i}}\left(\nabla_{i} F\right)^{2}
$$

and by Lemma 3.1 i)

$$
\nabla_{i} F=0, \quad \text { for } 1<i \leq \mu .
$$

From (3.5) and (3.7), we have

$$
J_{2}=\frac{\beta-1}{\beta} \kappa_{i}^{-2}\left(\nabla_{i} F\right)^{2}-\frac{2(\beta-1)}{\beta} \kappa_{i}^{-1} \frac{\partial \log F}{\partial \kappa_{i}}\left(\nabla_{i} F\right)^{2}+2 \frac{(\beta-1)^{2}}{\beta^{2}} \kappa_{1} \kappa_{i}^{-2} \frac{\partial \log F}{\partial \kappa_{i}}\left(\nabla_{i} F\right)^{2} .
$$

By Lemma 3.1 i) we also know

$$
\begin{aligned}
\frac{\partial^{2} F}{\partial h_{i j} \partial h_{s t}} h_{i j 1} h_{s t 1} & =\frac{\partial^{2} F}{\partial \kappa_{i} \partial \kappa_{j}} h_{i i 1} h_{j j 1}+2 \sum_{i>j}\left(\kappa_{i}-\kappa_{j}\right)^{-1}\left(\frac{\partial F}{\partial \kappa_{i}}-\frac{\partial F}{\partial \kappa_{j}}\right) h_{i j 1}^{2} \\
& =\frac{\partial^{2} F}{\partial \kappa_{i} \partial \kappa_{j}} h_{i i 1} h_{j j 1}+2 \sum_{i>\mu}\left(\kappa_{i}-\kappa_{1}\right)^{-1}\left(\frac{\partial F}{\partial \kappa_{i}}-\frac{\partial F}{\partial \kappa_{1}}\right) h_{11 i}^{2} \\
& +2 \sum_{i>j>\mu}\left(\kappa_{i}-\kappa_{j}\right)^{-1}\left(\frac{\partial F}{\partial \kappa_{i}}-\frac{\partial F}{\partial \kappa_{j}}\right) h_{i j 1}^{2}
\end{aligned}
$$

and

$$
\begin{aligned}
2 \frac{\partial F}{\partial \kappa_{i}} \sum_{l>\mu}\left(\kappa_{l}-\kappa_{1}\right)^{-1} h_{1 l i}^{2} & =2 \frac{\partial F}{\partial \kappa_{1}} \sum_{l>\mu}\left(\kappa_{l}-\kappa_{1}\right)^{-1} h_{11 l}^{2}+2 \sum_{i>\mu} \frac{\partial F}{\partial \kappa_{i}}\left(\kappa_{i}-\kappa_{1}\right)^{-1} h_{1 i i}^{2} \\
& +2 \sum_{i>l>\mu} \frac{\partial F}{\partial \kappa_{i}}\left(\kappa_{l}-\kappa_{1}\right)^{-1} h_{1 l i}^{2}+2 \sum_{l>i>\mu} \frac{\partial F}{\partial \kappa_{i}}\left(\kappa_{l}-\kappa_{1}\right)^{-1} h_{1 l i}^{2} .
\end{aligned}
$$


And

$$
\begin{aligned}
& 2 \sum_{i>j>\mu}\left(\kappa_{i}-\kappa_{j}\right)^{-1}\left(\frac{\partial F}{\partial \kappa_{i}}-\frac{\partial F}{\partial \kappa_{j}}\right) h_{i j 1}^{2}+2 \sum_{i>l>\mu} \frac{\partial F}{\partial \kappa_{i}}\left(\kappa_{l}-\kappa_{1}\right)^{-1} h_{1 l i}^{2}+2 \sum_{l>i>\mu} \frac{\partial F}{\partial \kappa_{i}}\left(\kappa_{l}-\kappa_{1}\right)^{-1} h_{1 l i}^{2} \\
\geq & 2 \sum_{i>j>\mu}\left(\kappa_{i}-\kappa_{j}\right)^{-1}\left(\frac{\partial F}{\partial \kappa_{i}}-\frac{\partial F}{\partial \kappa_{j}}\right) h_{i j 1}^{2}+2 \sum_{i>l>\mu} \frac{\partial F}{\partial \kappa_{i}} \kappa_{l}^{-1} h_{1 l i}^{2}+2 \sum_{l>i>\mu} \frac{\partial F}{\partial \kappa_{i}} \kappa_{l}^{-1} h_{1 l i}^{2} \\
= & 2 \sum_{i>j>\mu} \kappa_{i}^{-1} \kappa_{j}^{-1}\left(\kappa_{i}-\kappa_{j}\right)^{-1}\left(\frac{\partial F}{\partial \kappa_{i}} \kappa_{i}^{2}-\frac{\partial F}{\partial \kappa_{j}} \kappa_{j}^{2}\right) h_{i j 1}^{2} \geq 0,
\end{aligned}
$$

where the last inequality is due to Condition 1.1 iii).

Now we have

$$
\begin{aligned}
J_{3} & \geq F \kappa_{1}^{-2} \frac{\partial^{2} F}{\partial \kappa_{i} \partial \kappa_{j}} h_{i i 1} h_{j j 1}+2 F \kappa_{1}^{-2} \sum_{i>\mu}\left(\kappa_{i}-\kappa_{1}\right)^{-1} \frac{\partial F}{\partial \kappa_{i}} h_{11 i}^{2}+2 F \kappa_{1}^{-2} \sum_{i>\mu} \frac{\partial F}{\partial \kappa_{i}}\left(\kappa_{i}-\kappa_{1}\right)^{-1} h_{1 i i}^{2} \\
& \geq-F \kappa_{1}^{-2} \kappa_{i}^{-1} \frac{\partial F}{\partial \kappa_{i}} h_{i i 1}^{2}+\kappa_{1}^{-2}\left(\nabla_{1} F\right)^{2}+2 \kappa_{1}^{2} \sum_{i>\mu}\left(\kappa_{i}-\kappa_{1}\right)^{-1}\left(\kappa_{1}^{-1}-\frac{\beta-1}{\beta} \kappa_{i}^{-1}\right)^{2} \frac{\partial \log F}{\partial \kappa_{i}}\left(\nabla_{i} F\right)^{2} \\
& +2 F \kappa_{1}^{-2} \sum_{i>\mu} \frac{\partial F}{\partial \kappa_{i}}\left(\kappa_{i}-\kappa_{1}\right)^{-1} h_{1 i i}^{2} \\
& \geq-\frac{1}{\beta^{2}} \kappa_{1}^{-1} \frac{\partial \log F}{\partial \kappa_{1}}\left(\nabla_{1} F\right)^{2}+\kappa_{1}^{-2}\left(\nabla_{1} F\right)^{2}+2 \kappa_{1}^{2} \sum_{i>\mu}\left(\kappa_{i}-\kappa_{1}\right)^{-1}\left(\kappa_{1}^{-1}-\frac{\beta-1}{\beta} \kappa_{i}^{-1}\right)^{2} \frac{\partial \log F}{\partial \kappa_{i}}\left(\nabla_{i} F\right)^{2}
\end{aligned}
$$

where the second inequality is from Condition $1.1 \mathrm{iv}$ ) and (3.7), the last inequality is from

$$
-F \kappa_{1}^{-2} \kappa_{i}^{-1} \frac{\partial F}{\partial \kappa_{i}} h_{i i 1}^{2}+2 F \kappa_{1}^{-2} \sum_{i>\mu} \frac{\partial F}{\partial \kappa_{i}}\left(\kappa_{i}-\kappa_{1}\right)^{-1} h_{1 i i}^{2} \geq-F \kappa_{1}^{-3} \frac{\partial F}{\partial \kappa_{1}} h_{111}^{2}
$$

and

$$
-F \kappa_{1}^{-3} \frac{\partial F}{\partial \kappa_{1}} h_{111}^{2}=-\frac{1}{\beta^{2}} \kappa_{1}^{-1} \frac{\partial \log F}{\partial \kappa_{1}}\left(\nabla_{1} F\right)^{2} .
$$

Using (3.8), we have

$$
\begin{aligned}
J_{2}+J_{3} & \geq \frac{\beta-1}{\beta} \kappa_{i}^{-2}\left(\nabla_{i} F\right)^{2}-\frac{2(\beta-1)}{\beta} \kappa_{i}^{-1} \frac{\partial \log F}{\partial \kappa_{i}}\left(\nabla_{i} F\right)^{2}+2 \frac{(\beta-1)^{2}}{\beta^{2}} \kappa_{1} \kappa_{i}^{-2} \frac{\partial \log F}{\partial \kappa_{i}}\left(\nabla_{i} F\right)^{2} \\
& -\frac{1}{\beta^{2}} \kappa_{1}^{-1} \frac{\partial \log F}{\partial \kappa_{1}}\left(\nabla_{1} F\right)^{2}+\kappa_{1}^{-2}\left(\nabla_{1} F\right)^{2}+2 \kappa_{1}^{2} \sum_{i>\mu}\left(\kappa_{i}-\kappa_{1}\right)^{-1}\left(\kappa_{1}^{-1}-\frac{\beta-1}{\beta} \kappa_{i}^{-1}\right)^{2} \frac{\partial \log F}{\partial \kappa_{i}}\left(\nabla_{i} F\right)^{2} \\
& =\sum_{i>\mu}\left(\frac{\beta-1}{\beta} \kappa_{i}^{-2}+\frac{2}{\beta} \kappa_{1}\left(\kappa_{i}-\kappa_{1}\right)^{-1}\left(\kappa_{1}^{-1}-\frac{\beta-1}{\beta} \kappa_{i}^{-1}\right) \frac{\partial \log F}{\partial \kappa_{i}}\right)\left(\nabla_{i} F\right)^{2} \\
& +\frac{2 \beta-1}{\beta} \kappa_{1}^{-2}\left(1-\frac{1}{\beta} \frac{\partial \log F}{\partial \kappa_{1}} \kappa_{1}\right)\left(\nabla_{1} F\right)^{2} \geq 0 .
\end{aligned}
$$

And combining (3.3), we obtain

$$
0 \geq J_{1}
$$

On the other hand, since $\lambda^{\prime}(r)=\cos r \geq 0$ and $\epsilon=1$ for the hemishpere $\mathbb{S}_{+}^{n+1}$, by $\mathcal{F} \geq 0, \beta \geq 1$ and $\frac{\kappa_{i}}{\kappa_{1}} \geq 1$, we know $J_{1} \geq 0$. Thus, $J_{1}=0=J_{2}+J_{3}$, which implies $\kappa_{1}=\ldots=\kappa_{n}$ and $\nabla F=0$ at $\bar{x}$. 


\section{Proof of Theorem 1.2 When $\beta>1$}

Coming back to the test function $Z=F \operatorname{tr} b-\frac{n(\beta-1)}{\beta} \Phi$ and considering $\mathcal{L} Z$ according to (ii) in Corollary 2.2, we have

$$
\begin{aligned}
& 2 \frac{\partial F}{\partial h_{i j}} \nabla_{i} F \nabla_{j} \operatorname{tr} b+\bar{g}\left(\lambda \partial_{r}, \nabla(F \operatorname{tr} b)\right) \\
= & 2 F^{-1} \frac{\partial F}{\partial h_{i j}} \nabla_{i} F \nabla_{j}(F \operatorname{tr} b)-2 F^{-1} \operatorname{tr} b \frac{\partial F}{\partial h_{i j}} \nabla_{i} F \nabla_{j} F+\bar{g}\left(\lambda \partial_{r}, \nabla(F \operatorname{tr} b)\right) \\
= & 2 F^{-1} \frac{\partial F}{\partial h_{i j}} \nabla_{i} F \nabla_{j} Z+\frac{2 n(\beta-1)}{\beta} F^{-1} \frac{\partial F}{\partial h_{i j}} \nabla_{i} F \nabla_{j} \Phi-2 F^{-1} \operatorname{tr} b \frac{\partial F}{\partial h_{i j}} \nabla_{i} F \nabla_{j} F \\
+ & \bar{g}\left(\lambda \partial_{r}, \nabla Z\right)+\frac{n(\beta-1)}{\beta} \bar{g}\left(\lambda \partial_{r}, \nabla \Phi\right) .
\end{aligned}
$$

Using $\nabla_{i} \Phi=\kappa_{i}^{-1} \nabla_{i} F$ which follows from $\nabla_{i} \Phi=\bar{g}\left(\lambda \partial_{r}, e_{i}\right)$ and $\nabla_{i} F=\kappa_{i} \bar{g}\left(\lambda \partial_{r}, e_{i}\right)$, we get

$$
\begin{aligned}
& 2 \frac{\partial F}{\partial h_{i j}} \nabla_{i} F \nabla_{j} \operatorname{tr} b+\bar{g}\left(\lambda \partial_{r}, \nabla(F \operatorname{tr} b)\right) \\
= & R(\nabla Z)+\frac{2 n(\beta-1)}{\beta} \kappa_{i}^{-1} \frac{\partial \log F}{\partial \kappa_{i}}\left(\nabla_{i} F\right)^{2}-2 \operatorname{tr} b \frac{\partial \log F}{\partial \kappa_{i}}\left(\nabla_{i} F\right)^{2} \\
+ & \frac{n(\beta-1)}{\beta} \kappa_{i}^{-2}\left(\nabla_{i} F\right)^{2}
\end{aligned}
$$

where $R(\nabla Z)$ denotes the terms including $\nabla Z$.

Using Condition 1.1 iv), we know

$$
\begin{aligned}
& F b^{l p} b^{q l} \frac{\partial^{2} F}{\partial h_{i j} \partial h_{s t}} h_{i j p} h_{s t q} \\
= & F \kappa_{p}^{-2} \frac{\partial^{2} F}{\partial \kappa_{i} \partial \kappa_{j}} h_{i i p} h_{j j p}+F \kappa_{p}^{-2} \sum_{i \neq j}\left(\frac{\partial F}{\partial \kappa_{i}}-\frac{\partial F}{\partial \kappa_{j}}\right)\left(\kappa_{i}-\kappa_{j}\right)^{-1} h_{i j p}^{2} \\
\geq & -F \kappa_{p}^{-2} \kappa_{i}^{-1} \frac{\partial F}{\partial \kappa_{i}} h_{i i p}^{2}+\kappa_{p}^{-2}\left(\nabla_{p} F\right)^{2}+F \kappa_{p}^{-2} \sum_{i \neq j}\left(\frac{\partial F}{\partial \kappa_{i}}-\frac{\partial F}{\partial \kappa_{j}}\right)\left(\kappa_{i}-\kappa_{j}\right)^{-1} h_{i j p}^{2} .
\end{aligned}
$$

Then combining

$$
\begin{aligned}
& 2 F b^{k s} b^{p t} b^{k q} \frac{\partial F}{\partial h_{i j}} h_{s t i} h_{p q j}=2 F \frac{\partial F}{\partial \kappa_{i}} \kappa_{p}^{-2} \kappa_{q}^{-1} h_{p q i}^{2} \\
= & 2 F \frac{\partial F}{\partial \kappa_{i}} \kappa_{p}^{-2} \kappa_{i}^{-1} h_{p i i}^{2}+2 F \sum_{q \neq i} \frac{\partial F}{\partial \kappa_{i}} \kappa_{p}^{-2} \kappa_{q}^{-1} h_{p q i}^{2},
\end{aligned}
$$


we get

$$
\begin{aligned}
& F b^{l p} b^{q l} \frac{\partial^{2} F}{\partial h_{i j} \partial h_{s t}} h_{i j p} h_{s t q}+2 F b^{k s} b^{p t} b^{k q} \frac{\partial F}{\partial h_{i j}} h_{s t i} h_{p q j} \\
\geq & F \kappa_{p}^{-2} \kappa_{i}^{-1} \frac{\partial F}{\partial \kappa_{i}} h_{i i p}^{2}+\kappa_{p}^{-2}\left(\nabla_{p} F\right)^{2}+F \kappa_{p}^{-2} \sum_{i \neq j}\left(\frac{\partial F}{\partial \kappa_{i}}-\frac{\partial F}{\partial \kappa_{j}}\right)\left(\kappa_{i}-\kappa_{j}\right)^{-1} h_{i j p}^{2} \\
+ & 2 F \sum_{q \neq i} \frac{\partial F}{\partial \kappa_{i}} \kappa_{p}^{-2} \kappa_{q}^{-1} h_{p q i}^{2} .
\end{aligned}
$$

By Condition 1.1 iii), we have

$$
\begin{aligned}
& F \kappa_{p}^{-2} \sum_{i \neq j}\left(\frac{\partial F}{\partial \kappa_{i}}-\frac{\partial F}{\partial \kappa_{j}}\right)\left(\kappa_{i}-\kappa_{j}\right)^{-1} h_{i j p}^{2}+2 F \sum_{q \neq i} \frac{\partial F}{\partial \kappa_{i}} \kappa_{p}^{-2} \kappa_{q}^{-1} h_{p q i}^{2} \\
= & F \kappa_{p}^{-2} \sum_{i \neq j}\left(\frac{\partial F}{\partial \kappa_{i}} \kappa_{i}^{2}-\frac{\partial F}{\partial \kappa_{j}} \kappa_{j}^{2}\right) \kappa_{i}^{-1} \kappa_{j}^{-1}\left(\kappa_{i}-\kappa_{j}\right)^{-1} h_{i j p}^{2} \geq 0 .
\end{aligned}
$$

According to the Cauchy-Schwartz inequality and $\sum_{i} \frac{\partial F}{\partial \kappa_{i}} \kappa_{i}=\beta F$, we have

$$
F \kappa_{p}^{-2} \kappa_{i}^{-1} \frac{\partial F}{\partial \kappa_{i}} h_{i i p}^{2} \geq \frac{1}{\beta} \kappa_{p}^{-2}\left(\nabla_{p} F\right)^{2} .
$$

Combining (4.2), (4.3) and (4.4), we know

$$
F b^{l p} b^{q l} \frac{\partial^{2} F}{\partial h_{i j} \partial h_{s t}} h_{i j p} h_{s t q}+2 F b^{k s} b^{p t} b^{k q} \frac{\partial F}{\partial h_{i j}} h_{s t i} h_{p q j} \geq \frac{\beta+1}{\beta} \kappa_{p}^{-2}\left(\nabla_{p} F\right)^{2} .
$$

For convenience, let us denote

$$
L_{1}=\epsilon F\left(\beta F \operatorname{tr}\left(b^{2}\right)-\operatorname{tr} b \sum_{i} \frac{\partial F}{\partial h_{i i}}\right)+(\beta-1) \lambda^{\prime}\left(F \operatorname{tr} b-\frac{n}{\beta} \sum_{i} \frac{\partial F}{\partial h_{i i}}\right)+\mathcal{F}\left(\operatorname{tr} b \frac{\partial F}{\partial h_{i j}} h_{i l} h_{j l}-n \beta F\right)
$$

It follows from Condition 1.1 iii) that

$$
\begin{aligned}
L_{1}= & \epsilon F \sum_{i>j} \frac{1}{\kappa_{i}^{2} \kappa_{j}^{2}}\left(\frac{\partial F}{\partial \kappa_{i}} \kappa_{i}^{2}-\frac{\partial F}{\partial \kappa_{j}} \kappa_{j}^{2}\right)\left(\kappa_{i}-\kappa_{j}\right)+\frac{(\beta-1)}{\beta} \lambda^{\prime} \sum_{i>j} \frac{1}{\kappa_{i} \kappa_{j}}\left(\frac{\partial F}{\partial \kappa_{i}} \kappa_{i}-\frac{\partial F}{\partial \kappa_{j}} \kappa_{j}\right)\left(\kappa_{i}-\kappa_{j}\right) \\
& +\mathcal{F} \sum_{i>j} \frac{1}{\kappa_{i} \kappa_{j}}\left(\frac{\partial F}{\partial \kappa_{i}} \kappa_{i}^{2}-\frac{\partial F}{\partial \kappa_{j}} \kappa_{j}^{2}\right)\left(\kappa_{i}-\kappa_{j}\right) \geq 0,
\end{aligned}
$$

and the equality holds if and only if $\kappa_{1}=\cdots=\kappa_{n}$. Thus adding (4.1), (4.5) and (4.7), we obtain

$$
\begin{aligned}
\mathcal{L} Z+R(\nabla Z) & \geq \frac{2 n(\beta-1)}{\beta} \kappa_{i}^{-1} \frac{\partial \log F}{\partial \kappa_{i}}\left(\nabla_{i} F\right)^{2}-2 \operatorname{tr} b \frac{\partial \log F}{\partial \kappa_{i}}\left(\nabla_{i} F\right)^{2} \\
& +\frac{n(\beta-1)}{\beta} \kappa_{i}^{-2}\left(\nabla_{i} F\right)^{2}+\frac{\beta+1}{\beta} \kappa_{i}^{-2}\left(\nabla_{i} F\right)^{2} \\
& =\left(2 \frac{\partial \log F}{\partial \kappa_{i}}\left(n \kappa_{i}^{-1}-\operatorname{tr} b\right)-\frac{2 n}{\beta} \kappa_{i}^{-1} \frac{\partial \log F}{\partial \kappa_{i}}+\frac{(n+1) \beta-n+1}{\beta} \kappa_{i}^{-2}\right)\left(\nabla_{i} F\right)^{2} .
\end{aligned}
$$


By Lemma 3.2, we know that any maximum point $\bar{x}$ of $W$ is an umbilic point on $M$. Thus at $\bar{x}$, we have

$$
\begin{aligned}
& 2 \frac{\partial \log F}{\partial \kappa_{i}}\left(n \kappa_{i}^{-1}-\operatorname{tr} b\right)-\frac{2 n}{\beta} \kappa_{i}^{-1} \frac{\partial \log F}{\partial \kappa_{i}}+\frac{(n+1) \beta-n+1}{\beta} \kappa_{i}^{-2} \\
= & \frac{(n-1)(\beta-1)}{\beta} \kappa_{i}^{-2}>0
\end{aligned}
$$

for any $1 \leq i \leq n$. Then there exists a neighborhood of $\bar{x}$, denoted by $U$, such that $\mathcal{L} Z+R(\nabla Z) \geq 0$ in $U$. Since $Z \leq n W \leq n W(\bar{x})=Z(\bar{x}), Z$ attains its maximum at $\bar{x}$. By the strong maximum principle, we know $Z=Z(\bar{x})$ is a constant in $U$, which implies $W$ is also a constant in $U$. Hence the set of maximum points of $W$ is an open set. Due to the connectedness of $M, W$ is a constant on $M$.

Then by Lemma 3.2, we know $\nabla F=0$ on $M$. From $\nabla_{i} F=\kappa_{i} \bar{g}\left(\lambda \partial_{r}, e_{i}\right)$ and $\kappa_{i}>0$, we know $\nu$ is parallel to $\partial_{r}$ at every point of $M$, which implies $M$ is a slice $\left\{r_{0}\right\} \times \mathbb{S}^{n}$. This completes the proof of Theorem 1.2 for $\beta>1$.

\section{Proof of Theorem 1.2 When $\beta=1$}

Notice that (4.8) vanishes for $\beta=1$, we need a new approach. The proof is divided into two cases according to the dimension of $M$.

5.1. For $n \geq 3$. When $\beta=1$, from (4.1), (4.2) and (4.7) we get

$$
\begin{aligned}
\mathcal{L} Z+R(\nabla Z) \geq & -2 \operatorname{tr} b \frac{\partial \log F}{\partial \kappa_{i}}\left(\nabla_{i} F\right)^{2}-F \kappa_{p}^{-2} \kappa_{i}^{-1} \frac{\partial F}{\partial \kappa_{i}} h_{i i p}^{2}+\kappa_{p}^{-2}\left(\nabla_{p} F\right)^{2} \\
& +F \kappa_{p}^{-2} \sum_{i \neq j}\left(\frac{\partial F}{\partial \kappa_{i}}-\frac{\partial F}{\partial \kappa_{j}}\right)\left(\kappa_{i}-\kappa_{j}\right)^{-1} h_{i j p}^{2}+2 F \frac{\partial F}{\partial \kappa_{i}} \kappa_{p}^{-2} \kappa_{q}^{-1} h_{p q i}^{2} .
\end{aligned}
$$

Notice now our test function is $Z=F \operatorname{tr} b$. Using

$$
-\kappa_{p}^{-2} h_{p p i}=\nabla_{j} \operatorname{tr} b=\operatorname{tr} b \nabla_{j} \log Z-\operatorname{tr} b \nabla_{j} \log F,
$$

we have

$$
\begin{aligned}
& 2 F^{2} \frac{\partial \log F}{\partial \kappa_{i}}\left(-\operatorname{tr} b\left(\nabla_{i} \log F\right)^{2}+\kappa_{p}^{-2} \kappa_{q}^{-1} h_{p q i}^{2}\right) \\
= & 2 F^{2} \frac{\partial \log F}{\partial \kappa_{i}}\left(\sum_{p} \kappa_{p}^{-1}\left(\kappa_{p}^{-2} h_{p p i}^{2}-\left(\nabla_{i} \log F\right)^{2}\right)+\sum_{p \neq q} \kappa_{p}^{-2} \kappa_{q}^{-1} h_{p q i}^{2}\right) \\
= & 2 F^{2} \frac{\partial \log F}{\partial \kappa_{i}}\left(\sum_{p} \kappa_{p}^{-1}\left(\kappa_{p}^{-1} h_{p p i}-\nabla_{i} \log F\right)^{2}+\sum_{p \neq q} \kappa_{p}^{-2} \kappa_{q}^{-1} h_{p q i}^{2}+R(\nabla Z)\right) .
\end{aligned}
$$


Moreover, we have

$$
\begin{aligned}
& 2 F^{2} \sum_{i} \sum_{p \neq q} \frac{\partial \log F}{\partial \kappa_{i}} \kappa_{p}^{-2} \kappa_{q}^{-1} h_{p q i}^{2}+F \sum_{p} \sum_{i \neq j} \kappa_{p}^{-2}\left(\frac{\partial F}{\partial \kappa_{i}}-\frac{\partial F}{\partial \kappa_{j}}\right)\left(\kappa_{i}-\kappa_{j}\right)^{-1} h_{i j p}^{2} \\
& =2 F^{2} \sum_{i \neq p} \frac{\partial \log F}{\partial \kappa_{i}} \kappa_{p}^{-2} \kappa_{i}^{-1} h_{p i i}^{2}+F \sum_{\neq} \frac{\frac{\partial F}{\partial \kappa_{i}} \kappa_{i}^{2}-\frac{\partial F}{\partial \kappa_{j}} \kappa_{j}^{2}}{\kappa_{i} \kappa_{j}\left(\kappa_{i}-\kappa_{j}\right)} \kappa_{p}^{-2} h_{i j p}^{2} \\
& +2 F \sum_{i \neq p} \frac{\frac{\partial F}{\partial \kappa_{i}} \kappa_{i}-\frac{\partial F}{\partial \kappa_{p}} \kappa_{p}}{\kappa_{i}-\kappa_{p}} \kappa_{p}^{-2} \kappa_{i}^{-1} h_{i p p}^{2} \\
& \geq 2 F^{2} \sum_{i \neq p} \frac{\partial \log F}{\partial \kappa_{i}} \kappa_{p}^{-2} \kappa_{i}^{-1} h_{p i i}^{2}
\end{aligned}
$$

where $\sum_{\neq}$denotes that the three summation indices are distinct. Thus we know

$$
\begin{aligned}
\mathcal{L} Z+R(\nabla Z) \geq & 2 F^{2} \frac{\partial \log F}{\partial \kappa_{i}} \kappa_{p}^{-1}\left(\kappa_{p}^{-1} h_{p p i}-\nabla_{i} \log F\right)^{2}-F \kappa_{p}^{-2} \kappa_{i}^{-1} \frac{\partial F}{\partial \kappa_{i}} h_{i i p}^{2} \\
& +\kappa_{p}^{-2}\left(\nabla_{p} F\right)^{2}+2 F^{2} \sum_{i \neq p} \frac{\partial \log F}{\partial \kappa_{i}} \kappa_{p}^{-2} \kappa_{i}^{-1} h_{p i i}^{2} \\
& =2 F^{2} \sum_{i \neq p} \frac{\partial \log F}{\partial \kappa_{i}} \kappa_{p}^{-1}\left(\kappa_{p}^{-1} h_{p p i}-\nabla_{i} \log F\right)^{2}+F \kappa_{p}^{-2} \kappa_{i}^{-1} \frac{\partial F}{\partial \kappa_{i}} h_{i i p}^{2} \\
& +\kappa_{p}^{-2}\left(\nabla_{p} F\right)^{2}+2 F^{2} \frac{\partial \log F}{\partial \kappa_{i}} \kappa_{i}^{-1}\left(\nabla_{i} \log F\right)^{2} \\
& -4 F^{2} \frac{\partial \log F}{\partial \kappa_{i}} \kappa_{i}^{-2} h_{i i i} \nabla_{i} \log F .
\end{aligned}
$$

Let $y_{p}=\frac{\partial \log F}{\partial \kappa_{p}} h_{p p i}$ and $t_{i}=\frac{\partial \log F}{\partial \kappa_{i}} \kappa_{i}$, then

$$
\kappa_{p}^{-2}\left(\nabla_{p} F\right)^{2}+2 F^{2} \frac{\partial \log F}{\partial \kappa_{i}} \kappa_{i}^{-1}\left(\nabla_{i} \log F\right)^{2}=F^{2} \kappa_{i}^{-2}\left(1+2 t_{i}\right)\left(\sum_{p} y_{p}\right)^{2}
$$

and

$$
\begin{aligned}
& F \kappa_{p}^{-2} \kappa_{i}^{-1} \frac{\partial F}{\partial \kappa_{i}} h_{i i p}^{2}-4 F^{2} \frac{\partial \log F}{\partial \kappa_{i}} \kappa_{i}^{-2} h_{i i i} \nabla_{i} \log F \\
= & F^{2} \kappa_{i}^{-2} \sum_{p}\left(\kappa_{p}^{-1} \frac{\partial \log F}{\partial \kappa_{p}} h_{p p i}^{2}-4 \frac{\partial \log F}{\partial \kappa_{i}} h_{i i i} \frac{\partial \log F}{\partial \kappa_{p}} h_{p p i}\right) \\
= & F^{2} \kappa_{i}^{-2} \sum_{p}\left(\frac{1}{t_{p}} y_{p}^{2}-4 y_{i} y_{p}\right) .
\end{aligned}
$$

Therefore

$$
\mathcal{L} Z+R(\nabla Z) \geq F^{2} \kappa_{i}^{-2}\left(\sum_{p}\left(\frac{1}{t_{p}} y_{p}^{2}-4 y_{i} y_{p}\right)+\left(1+2 t_{i}\right)\left(\sum_{p} y_{p}\right)^{2}\right) .
$$

Since $\sum_{i=1}^{n} t_{i}=1$ for $\beta=1$ and $t_{i}>0$ for any $i$, using Lagrangian multiplier technique, we have $\sum_{p}\left(\frac{1}{t_{p}} y_{p}^{2}-4 y_{i} y_{p}\right) \geq\left(1-8 t_{i}+4 t_{i}^{2}\right)\left(\sum_{p} y_{p}\right)^{2}$ (see Lemma 6.2 in 
[9]). Thus, we have

$$
\mathcal{L} Z+R(\nabla Z) \geq 2 F^{2} \kappa_{i}^{-2}\left(1-3 t_{i}+2 t_{i}^{2}\right)\left(\sum_{p} y_{p}\right)^{2} .
$$

It follows from $\sum_{i=1}^{n} t_{i}=1$ that $t_{i}=\frac{1}{n}$ at any umbilic point for each $i$. Thus

$$
1-3 t_{i}+2 t_{i}^{2}=\frac{(n-1)(n-2)}{n^{2}}>0
$$

at an umbilic point if $n \geq 3$. For $n \geq 3$, the rest of the proof is as same as the one for $\beta>1$ in Section 4 by using Lemma 3.2.

5.2. For $n=2$. In the case of $n=2$, notice that $1-3 t_{i}+2 t_{i}^{2}=0$ in (5.2). So instead of the above argument, we will show directly that $\mathcal{L} Z+R(\nabla Z) \geq 0$ at all point of $M$. We have known this holds at umbilic points from (5.2). Thus we assume $\kappa_{1} \neq \kappa_{2}$ below.

We know

$$
\nabla_{i} \operatorname{tr} b=-\kappa_{1}^{-2} h_{11 i}-\kappa_{2}^{-2} h_{22 i}=-t_{1}^{-1} \kappa_{1}^{-1} y_{1}-t_{2}^{-1} \kappa_{2}^{-1} y_{2}
$$

and

$$
-\operatorname{tr} b \nabla_{i} \log F=-\left(\kappa_{1}^{-1}+\kappa_{2}^{-1}\right)\left(y_{1}+y_{2}\right) .
$$

By

$$
\nabla_{j} \operatorname{tr} b=R(\nabla Z)-\operatorname{tr} b \nabla_{j} \log F
$$

we have

$$
t_{1}^{-1} \kappa_{1}^{-1} y_{1}+t_{2}^{-1} \kappa_{2}^{-1} y_{2}=\left(\kappa_{1}^{-1}+\kappa_{2}^{-1}\right)\left(y_{1}+y_{2}\right)+R(\nabla Z) .
$$

Multiplying $t_{1} t_{2}$ on both sides and using $t_{1}+t_{2}=1$, we see

$$
t_{2}\left(t_{1}+t_{2}\right) \kappa_{1}^{-1} y_{1}+t_{1}\left(t_{1}+t_{2}\right) \kappa_{2}^{-1} y_{2}=t_{1} t_{2}\left(\kappa_{1}^{-1}+\kappa_{2}^{-1}\right)\left(y_{1}+y_{2}\right)+R(\nabla Z) .
$$

This implies

$$
t_{2}\left(t_{2} \kappa_{1}^{-1}-t_{1} \kappa_{2}^{-1}\right) y_{1}=t_{1}\left(t_{2} \kappa_{1}^{-1}-t_{1} \kappa_{2}^{-1}\right) y_{2}+R(\nabla Z),
$$

which means

$$
t_{2} y_{1}=t_{1} y_{2}+R(\nabla Z)
$$

or equivalently

$$
y_{1}=t_{1}\left(y_{1}+y_{2}\right)+R(\nabla Z)
$$

From (5.1), we have

$$
\mathcal{L} Z+R(\nabla Z) \geq F^{2} \kappa_{i}^{-2}\left(t_{1}^{-1} y_{1}^{2}+t_{2}^{-1} y_{2}^{2}-4 y_{i}\left(y_{1}+y_{2}\right)+\left(1+2 t_{i}\right)\left(y_{1}+y_{2}\right)^{2}\right) .
$$

By using (5.3) for $i=1$, similarly for $i=2$ as well, we have

$$
\begin{aligned}
& t_{1}^{-1} y_{1}^{2}+t_{2}^{-1} y_{2}^{2}-4 y_{1}\left(y_{1}+y_{2}\right)+\left(1+2 t_{1}\right)\left(y_{1}+y_{2}\right)^{2} \\
= & t_{1}^{-1} y_{1}^{2}+t_{2}^{-1} y_{2}^{2}-4 t_{1}^{-1} y_{1}^{2}+\left(1+2 t_{1}\right) t_{1}^{-2} y_{1}^{2}+R(\nabla Z) \\
= & t_{2}^{-1} y_{2}^{2}+\left(1-t_{1}\right) t_{1}^{-2} y_{1}^{2}+R(\nabla Z)=t_{2}^{-1} y_{2}^{2}+t_{2} t_{1}^{-2} y_{1}^{2}+R(\nabla Z) .
\end{aligned}
$$

Then we know

$$
\mathcal{L} Z+R(\nabla Z) \geq 0
$$


By the strong maximum principle, we know $Z$ is a constant. Then $\epsilon$-term vanishes which implies $M$ is totally umbilic. This also means that $W$ is a constant on $M$. As the discussion in Section 4, we finish the proof.

\section{Proof of Corollary 1.6 and Theorem 1.8}

Proof of Corollary 1.6. From Theorem 1.2, we know Corollary 1.6 is established for $\alpha \geq \frac{1}{n}$. When $\frac{1}{n+2} \leq \alpha<\frac{1}{n}$, it can be proven in a similar way as the Euclidean case (Refer to [5] for $\mathcal{F}=0$ and [9] for $\mathcal{F}>0$ ). Compared to the Euclidean space, the only different terms in equation $(i i)$ of Corollary 2.2 for $\mathcal{L} Z$ are

$$
(\beta-1) \lambda^{\prime}\left(F \operatorname{tr} b-\frac{n}{\beta} \sum_{i} \frac{\partial F}{\partial h_{i i}}\right)+\epsilon F\left(\beta F \operatorname{tr}\left(b^{2}\right)-\operatorname{tr} b \sum_{i} \frac{\partial F}{\partial h_{i i}}\right) .
$$

Observe that

$$
F \operatorname{tr} b-\frac{n}{\beta} \sum_{i} \frac{\partial F}{\partial h_{i i}}=0
$$

for $F=\sigma_{n}^{\alpha}$. And by Lemma 2.3, we know the $\epsilon$-terms with $\epsilon=1$ is nonnegative. Using the similar argument of the Euclidean case (see $[5,9]$ ), we know $Z$ is a constant. Thus the $\epsilon$-term must vanishes, which implies $M$ is totally umbilic by Lemma 2.3. It also means that $W$ is a constant on $M$. Thus the proof can be completed by the same method employed in Section 4 .

Proof of Theorem 1.8. In the hyperbolic space $\mathbb{H}^{3}, \lambda^{\prime}(r)=\cosh r>0$, under the assumption, it is easy to check $J_{1} \geq 0$ in (3.4) and the equality occurs if and only if $\kappa_{1}=\kappa_{2}$. Therefore Lemma 3.2 is established for this case. Taking $n=2, \epsilon=-1$, $F=\sigma_{2}$ and $\mathcal{F} \geq 1$ into consideration, we know

$$
\begin{aligned}
& \epsilon\left(\beta F^{2} \operatorname{tr}\left(b^{2}\right)-F \operatorname{tr} b \sum_{i} \frac{\partial F}{\partial h_{i i}}\right)+\mathcal{F}\left(\operatorname{tr} b \frac{\partial F}{\partial h_{i j}} h_{i l} h_{j l}-n \beta F\right) \\
= & -\left(2 \sigma_{2}^{2} \operatorname{tr}\left(b^{2}\right)-\sigma_{1} \sigma_{2} \operatorname{tr} b\right)+\mathcal{F}\left(\sigma_{1} \sigma_{2} \operatorname{tr} b-4 \sigma_{2}\right) \\
= & (\mathcal{F}-1)\left(\kappa_{1}-\kappa_{2}\right)^{2} \geq 0
\end{aligned}
$$

and

$$
(\beta-1) \lambda^{\prime}\left(F \operatorname{tr} b-\frac{n}{\beta} \sum_{i} \frac{\partial F}{\partial h_{i i}}\right)=0 .
$$

This leads to $L_{1} \geq 0$ in (4.6). Thus using the same argument as in Section 4 , we can easily carry out the proof of this theorem.

\section{REFERENCES}

[1] L. J. Alías, J. H. de Lira and M. Rigoli, Mean curvature flow solitons in the presence of conformal vector fields, 2017, arXiv:1707.07132v1.

[2] B. Andrews, Contraction of convex hypersurfaces by their affine normal, J Differential Geom, 1996, 43: 207-230.

[3] B. Andrews B and X. Chen, Curvature flow in hyperbolic spaces, J Reine Angew Math, 2017, 729: 29-49.

[4] B. Andrews, P.-F. Guan and L. Ni, Flow by powers of the Gauss curvature, Adv Math, 2016, 299: $174-201$.

[5] S. Brendle, K. Choi and P. Daskalopoulos, Asymptotic behavior of flows by powers of the Gaussian curvature, 2016, arXiv:1610.08933.

[6] K. Choi and P. Daskalopoulos, Uniqueness of closed self-similar solutions to the Gauss curvature flow, 2016, arXiv:1609.05487. 
[7] T. H. Colding and W. P. Minicozzi II, Generic mean curvature flow I: generic singularities, Annals of Mathematics, 2012, 2(2): 755-833.

[8] A. Futaki, K. Hattori and H. Yamamoto, Self-similar solutions to the mean curvature flows on Riemannian cone manifolds and special Lagrangians on toric Calabi-Yau cones, Osaka J. Math., 2014, 51: 1053-1079.

[9] S. Z. Gao, H. Li and H. Ma, Uniqueness of closed self-similar solutions to $\sigma_{k}^{\alpha}$-curvature flow, 2017, arXiv:1701.02642v2.

[10] P.-F. Guan and L. Ni, Entropy and a convergence theorem for Gauss curvature flow in high dimensions, J. Eur. Math. Soc., 2017, 19 (12): 3735-3761.

[11] G. Huisken, Asymptotic behavior for singularities of the mean curvature flow, J. Differential Geom., 1990, 31: 285-299.

[12] J. A. McCoy, Self-similar solutions of fully nonlinear curvature flows, Ann. Sc. Norm. Super Pisa Cl. Sci., 2011, 10 (5): 317-333.

[13] B. O'Neill, Semi-Riemannian geometry with applications to relativity, Pure and applied mathematics, vol. 103, Academic Press, London, 1983.

[14] G. Wu, The self-shrinker in warped product space and the weighted Minkowski inequality, Proc. Amer. Math. Soc., 2017, 145 (4): 1763-1772.

Department of Mathematical Sciences, Tsinghua University, Beijing 100084, P.R. CHINA;

E-mail address: gsz15@mails.tsinghua.edu.cn,hma@math.tsinghua.edu.cn 